\title{
Molecular diagnosis to identify new sources of resistance to sclerotinia blight in groundnut (Arachis hypogaea $\mathbf{L}$.)
}

\author{
Engin Yol $\cdot$ Hari D. Upadhyaya $\cdot$ Bulent Uzun
}

Received: 11 August 2014/ Accepted: 15 October 2014/Published online: 7 November 2014

(C) Springer Science+Business Media Dordrecht 2014

\begin{abstract}
Sclerotinia blight, caused by soil-borne fungus Sclerotinia minor Jagger, is one of the destructive diseases in groundnut. Pathogen affected plants usually displays lesions, wilt and collapse which cause high yield losses. Traditional field screening is time and resources consuming. Molecular markers associated with resistance genes offer an alternative selection technique which is relatively easy, more definite and not influenced by environmental fluctuations. In the present investigation, a marker-assisted diagnosis was done to screen 256 diverse germplasm for the presence or absence of SSR markers reported resistance or susceptibility to sclerotinia blight. One hundred and forty two genotypes from different botanical varieties were recognized as new potential sources of resistance to sclerotinia blight for field evaluation. The banding pattern related to the disease resistance is observed at high frequency in the variety vulgaris $(39.4 \%)$ and less distributed in the varieties fastigiata $(38.0 \%)$ and hypogaea $(19.7 \%)$ among the resistant genotypes in the collection. These genotypes had same banding pattern as reported for resistance germplasm. This work reports the
\end{abstract}

E. Yol · B. Uzun $(\bowtie)$

Department of Field Crops, Faculty of Agriculture, Akdeniz University, 07058 Antalya, Turkey

e-mail: bulentuzun@akdeniz.edu.tr

H. D. Upadhyaya

International Crops Research Institute for the Semi-Arid

Tropics (ICRISAT), Hyderabad, India successful application of marker-assisted diagnosis as a tool to identify resistance to sclerotinia blight in diverse collections.

Keywords Sclerotinia minor Jagger · Disease resistance $\cdot$ Pathogen $\cdot$ Molecular markers . Marker-assisted selection

\section{Introduction}

Groundnut (Arachis hypogaea L., Fabaceae), also known as peanut, is one of the major oilseed crops worldwide in tropical, subtropical and warm areas of approximately 100 countries, grown on 24.7 million ha with a total production of nearly 41 million tons annually (FAO 2012). About two-thirds of global production is used for vegetable oil and the remaining is utilized for edible product and as seed (Upadhyaya et al. 2011). Groundnut is a rich source of edible oil (about $48 \%$ ) and protein (about $26 \%$ ), used globally for human nutrition (Sarvamangala et al. 2011). The biological value of groundnut protein is among the highest of the vegetable proteins (Shoba et al. 2012). The cake obtained after extraction of oil and plant haulms are used in the livestock feed industry (Nigam and Aruna 2008). Groundnut also fixes atmospheric nitrogen, thus, improving the soil fertility.

Many biotic and abiotic stress factors limit the groundnut production in various eco-agricultural 
systems. Leaf spots, rust and soil-borne diseases among the biotic stresses are globally important diseases affecting both production and seed quality (Liao and Holbrook 2007). Of recent, soil-borne pathogens have become more important in many parts of the world (Thiessen and Woodward 2012). Sclerotinia blight is one of the most destructive soil-borne diseases of groundnut (Livingstone et al. 2005), causing yield losses up to $50 \%$ (Butzler et al. 1998). Sclerotinia blight is caused by soil-borne fungus $S$. minor Jagger which is an ascomycetes that produce white aerial mycelia and black, irregularly shaped sclerotia (Thiessen and Woodward 2012). Soil with $\mathrm{pH}$ near 6.5, cool temperatures and high relative humidity or rainfall are the favorable conditions for sclerotial germination and infection (Bailey and Brune 1997). Pathogens usually attack groundnut root and stem at or near the soil surface and destroy the vascular tissue of the crown, at which time the plant wilts and collapses (Laemmlen 2001). Several fungicides have been recommended to control the disease (Smith et al. 1992). Cultural practices such as crop rotation (Melouk and Backman 1995), tillage practices (Wu and Subbarao 2003), seed fungicide-treatment (Porter and Melouk 1997), and drip irrigation have been used to reduce the production costs, and soil contamination by fungicides (Gil et al. 2008) but these measures have not been sufficient to disease control. Host plant resistance is the most effective solution to manage sclerotinia blight and protect the environment (Chenault et al. 2009). However, desired progress through genetic resistance has not been achieved because of complicated inheritance mechanism and limited number of known sources of resistance (Chamberlin et al. 2010). Screening of more germplasm might help to find new sources of resistance to soil borne diseases to support breeding programs.

Traditional method of screening of germplasm in affected field plots is time and resource consuming. Several factors contribute to the development of uniform occurrence of diseases in the field conditions, which often make it difficult to achieve uniform infestation of disease pressure on test genotypes, leading to misclassification of germplasm. Advances in molecular marker technologies have opened the door to applying these techniques for screening of breeding populations to increase the efficiency of selection (Boopathi 2013). Marker assisted diagnosis probably would be cost-effective and faster for selection of resistance vis-à-vis field evaluations (Arus and Moreno-Gonzalez 1993; Ender et al. 2008; Ashkani et al. 2012). Simple sequence repeats (SSRs) are the marker of choice for use in such applications as these are reproducible and co-dominant markers used extensively in breeding studies (Mondal and Badigannavar 2010). Simple sequence repeats in groundnut have been used to identify resistance to late leaf spot (Shoba et al. 2012), rust (Mace et al. 2006), and nematode (Nagy et al. 2010). Simple sequence repeats associated with resistance to sclerotinia blight have been reported in groundnut (Chenault et al. 2009), which were used to characterize U.S. peanut mini core collection (Chamberlin et al. 2010) to identify sclerotinia blight resistant germplasm.

The aim of this investigation was to identify new sources of variation for sclerotinia blight in 256 peanut germplasm using SSR markers previously reported associated with resistance to sclerotinia blight.

\section{Materials and methods}

\section{Plant materials}

Two hundred and fifty six groundnut germplasm, which include ICRISAT groundnut mini core collection (Upadhyaya et al. 2002), breeding lines, local landraces, and registered cultivars, were used as a genetic material in this study (Table 1). PI 482189 (resistant) and PI 496448 (susceptible) were used as controls by Chamberlin et al. (2010) to differentiate the test materials into resistance or susceptible category based on SSR marker profile.

\section{Molecular analysis}

The seeds were germinated in the West Mediterranean Agricultural Research Institute's fields of Antalya, Turkey $\left(36^{\circ} 52^{\prime}\right.$ N. $30^{\circ} 50^{\prime}$ E. 15 m elevation). Groundnut leaves were collected from plants and stored at $-80{ }^{\circ} \mathrm{C}$ for DNA extraction. DNA isolation was carried out using the CTAB method (Doyle and Doyle 1990). The quality and quantity of the DNA extracts were checked by agarose gel electrophoresis with a DNA standard. The DNA extracts were suspended in milli-Q PCR water and stored at $-20{ }^{\circ} \mathrm{C}$.

The PCR analyses were conducted and templates for PCR reaction set up for $20 \mu \mathrm{l}$ as follows: $2 \mu \mathrm{l}$ of 
Table 1 Association of sclerotinia blight resistant marker with the genotypes of groundnut collection

\begin{tabular}{|c|c|c|c|c|c|c|c|c|c|}
\hline $\begin{array}{c}\text { Accession } \\
\text { No. }\end{array}$ & $\begin{array}{c}\text { ICRISAT } \\
\text { Genebank } \\
\text { Entry (ICG) / } \\
\text { Cultivar } \\
\text { Name }\end{array}$ & Subspecies & $\begin{array}{c}\text { Botanical } \\
\text { variety }\end{array}$ & $\begin{array}{l}\text { Marker } \\
\text { Score* }\end{array}$ & Accession No. & $\begin{array}{c}\text { ICRISAT } \\
\text { Genebank } \\
\text { Entry (ICG) / } \\
\text { Cultivar } \\
\text { Name }\end{array}$ & Subspecies & $\begin{array}{c}\text { Botanical } \\
\text { variety }\end{array}$ & $\begin{array}{c}\text { Marker } \\
\text { Score }\end{array}$ \\
\hline ACG 1 & ICG 36 & fastigiata & vulgaris & $\mathrm{L}$ & ACG 56 & ICG 4412 & hypogaea & hypogaea & $\mathrm{S}$ \\
\hline ACG 2 & ICG 76 & hypogaea & hypogaea & $\mathrm{S}$ & ACG 57 & ICG 4527 & hypogaea & hypogaea & $\mathrm{S}$ \\
\hline ACG 3 & ICG 81 & fastigiata & vulgaris & $\mathrm{L}$ & ACG 58 & ICG 4538 & hypogaea & hypogaea & $\mathrm{S}$ \\
\hline ACG 4 & ICG 111 & hypogaea & hypogaea & $\mathrm{S}$ & ACG 59 & ICG 4543 & fastigiata & vulgaris & $\mathrm{L}$ \\
\hline ACG 5 & ICG 115 & fastigiata & fastigiata & $\mathrm{L}$ & ACG 60 & ICG 4598 & hypogaea & hypogaea & $\mathrm{S}$ \\
\hline ACG 6 & ICG 118 & fastigiata & vulgaris & $\mathrm{S}$ & ACG 61 & ICG 4670 & fastigiata & fastigiata & $\mathrm{L}$ \\
\hline ACG 7 & ICG 156 & hypogaea & hypogaea & $\mathrm{S}$ & ACG 62 & ICG 4684 & fastigiata & vulgaris & $\mathrm{L}$ \\
\hline ACG 8 & ICG 163 & hypogaea & hypogaea & $\mathrm{S}$ & ACG 63 & ICG 4729 & fastigiata & vulgaris & $\mathrm{L}$ \\
\hline ACG 9 & ICG 188 & hypogaea & hypogaea & $\mathrm{L}$ & ACG 64 & ICG 4746 & hypogaea & hypogaea & $\mathrm{L}$ \\
\hline ACG 10 & ICG 297 & fastigiata & fastigiata & $\mathrm{L}$ & ACG 65 & ICG 4750 & fastigiata & vulgaris & $\mathrm{L}$ \\
\hline ACG 11 & ICG 332 & fastigiata & fastigiata & $\mathrm{L}$ & ACG 66 & ICG 4911 & fastigiata & vulgaris & $\mathrm{L}$ \\
\hline ACG 12 & ICG 334 & fastigiata & vulgaris & $\mathrm{L}$ & ACG 67 & ICG 4955 & fastigiata & vulgaris & $\mathrm{L}$ \\
\hline ACG 13 & ICG 397 & fastigiata & fastigiata & $\mathrm{L}$ & ACG 68 & ICG 4998 & hypogaea & hypogaea & $\mathrm{S}$ \\
\hline ACG 14 & ICG 434 & fastigiata & vulgaris & $\mathrm{L}$ & ACG 69 & ICG 5016 & hypogaea & hypogaea & $\mathrm{S}$ \\
\hline ACG 15 & ICG 442 & fastigiata & vulgaris & $\mathrm{L}$ & ACG 70 & ICG 5195 & fastigiata & vulgaris & $\mathrm{L}$ \\
\hline ACG 16 & ICG 513 & hypogaea & hypogaea & $\mathrm{S}$ & ACG 71 & ICG 5221 & fastigiata & fastigiata & $\mathrm{L}$ \\
\hline ACG 17 & ICG 532 & hypogaea & hypogaea & $\mathrm{S}$ & ACG 72 & ICG 5236 & fastigiata & vulgaris & $\mathrm{L}$ \\
\hline ACG 18 & ICG 721 & hypogaea & hypogaea & $\mathrm{S}$ & ACG 73 & ICG 5286 & hypogaea & hypogaea & $\mathrm{L}$ \\
\hline ACG 19 & ICG 862 & hypogaea & hypogaea & $\mathrm{S}$ & ACG 74 & ICG 5327 & hypogaea & hypogaea & $\mathrm{S}$ \\
\hline ACG 20 & ICG 875 & hypogaea & hypogaea & $\mathrm{S}$ & ACG 75 & ICG 5475 & fastigiata & fastigiata & $\mathrm{L}$ \\
\hline ACG 21 & ICG 928 & hypogaea & hypogaea & $\mathrm{S}$ & ACG 76 & ICG 5494 & fastigiata & vulgaris & $\mathrm{L}$ \\
\hline ACG 22 & ICG 1137 & fastigiata & vulgaris & $\mathrm{L}$ & ACG 77 & ICG 5609 & fastigiata & fastigiata & $\mathrm{L}$ \\
\hline ACG 23 & ICG 1142 & fastigiata & fastigiata & $\mathrm{L}$ & ACG 78 & ICG 5662 & hypogaea & hypogaea & $\mathrm{S}$ \\
\hline ACG 24 & ICG 1274 & fastigiata & fastigiata & $\mathrm{L}$ & ACG 79 & ICG 5663 & hypogaea & hypogaea & $\mathrm{S}$ \\
\hline ACG 25 & ICG 1399 & fastigiata & fastigiata & $\mathrm{L}$ & ACG 80 & ICG 5745 & hypogaea & hypogaea & $\mathrm{S}$ \\
\hline ACG 26 & ICG 1415 & fastigiata & vulgaris & $\mathrm{L}$ & ACG 81 & ICG 5779 & fastigiata & vulgaris & $\mathrm{L}$ \\
\hline ACG 27 & ICG 1519 & fastigiata & vulgaris & $\mathrm{L}$ & ACG 82 & ICG 5827 & hypogaea & hypogaea & $\mathrm{L}$ \\
\hline ACG 28 & ICG 1668 & hypogaea & hypogaea & $\mathrm{S}$ & ACG 83 & ICG 5891 & hypogaea & hypogaea & $\mathrm{S}$ \\
\hline ACG 29 & ICG 1711 & fastigiata & vulgaris & $\mathrm{L}$ & ACG 84 & ICG 6022 & fastigiata & fastigiata & $\mathrm{L}$ \\
\hline ACG 30 & ICG 1973 & fastigiata & vulgaris & $\mathrm{L}$ & ACG 85 & ICG 6057 & hypogaea & hypogaea & $\mathrm{S}$ \\
\hline ACG 31 & ICG 2019 & fastigiata & vulgaris & $\mathrm{L}$ & ACG 86 & ICG 6201 & fastigiata & fastigiata & $\mathrm{L}$ \\
\hline ACG 32 & ICG 2106 & fastigiata & vulgaris & $\mathrm{L}$ & ACG 87 & ICG 6263 & fastigiata & vulgaris & $\mathrm{L}$ \\
\hline ACG 33 & ICG 2381 & hypogaea & hypogaea & $\mathrm{L}$ & ACG 88 & ICG 6375 & fastigiata & vulgaris & $\mathrm{L}$ \\
\hline ACG 34 & ICG 2511 & hypogaea & hypogaea & $\mathrm{S}$ & ACG 89 & ICG 6402 & hypogaea & hypogaea & $\mathrm{L}$ \\
\hline ACG 35 & ICG 2738 & fastigiata & fastigiata & $\mathrm{L}$ & ACG 90 & ICG 6407 & fastigiata & vulgaris & $\mathrm{L}$ \\
\hline ACG 36 & ICG 2772 & hypogaea & hypogaea & $\mathrm{S}$ & ACG 91 & ICG 6646 & fastigiata & fastigiata & N/A \\
\hline ACG 37 & ICG 2773 & hypogaea & hypogaea & $\mathrm{L}$ & ACG 92 & ICG 6654 & fastigiata & vulgaris & N/A \\
\hline ACG 38 & ICG 2777 & hypogaea & hypogaea & $\mathrm{S}$ & ACG 93 & ICG 6667 & hypogaea & hypogaea & $\mathrm{S}$ \\
\hline ACG 39 & ICG 2857 & hypogaea & hypogaea & $\mathrm{L}$ & ACG 94 & ICG 6703 & hypogaea & hypogaea & $\mathrm{L}$ \\
\hline ACG 40 & ICG 2925 & hypogaea & hypogaea & $\mathrm{S}$ & ACG 95 & ICG 6766 & hypogaea & hypogaea & $\mathrm{S}$ \\
\hline $\mathrm{ACG} 41$ & ICG 3027 & hypogaea & hypogaea & $\mathrm{S}$ & ACG 96 & ICG 6813 & hypogaea & hypogaea & $\mathrm{S}$ \\
\hline $\mathrm{ACG} 42$ & ICG 3053 & hypogaea & hypogaea & $\mathrm{S}$ & ACG 97 & ICG 6888 & fastigiata & fastigiata & $\mathrm{L}$ \\
\hline ACG 43 & ICG 3102 & fastigiata & vulgaris & $\mathrm{L}$ & ACG 98 & ICG 6892 & hypogaea & hypogaea & $\mathrm{S}$ \\
\hline $\mathrm{ACG} 44$ & ICG 3240 & fastigiata & vulgaris & $\mathrm{L}$ & ACG 99 & ICG 6993 & hypogaea & hypogaea & $\mathrm{L}$ \\
\hline ACG 45 & ICG 3343 & fastigiata & vulgaris & $\mathrm{L}$ & ACG 100 & ICG 7000 & hypogaea & hypogaea & $\mathrm{L}$ \\
\hline ACG 46 & ICG 3421 & fastigiata & vulgaris & $\mathrm{L}$ & ACG 101 & ICG 7153 & hypogaea & hypogaea & $\mathrm{S}$ \\
\hline ACG 47 & ICG 3584 & fastigiata & vulgaris & $\mathrm{L}$ & ACG 102 & ICG 7181 & fastigiata & fastigiata & $\mathrm{L}$ \\
\hline $\mathrm{ACG} 48$ & ICG 3673 & fastigiata & fastigiata & $\mathrm{L}$ & ACG 103 & ICG 7190 & fastigiata & vulgaris & $\mathrm{L}$ \\
\hline ACG 49 & ICG 3681 & fastigiata & fastigiata & $\mathrm{L}$ & ACG 104 & ICG 7243 & hypogaea & hypogaea & $\mathrm{S}$ \\
\hline ACG 50 & ICG 3746 & fastigiata & vulgaris & $\mathrm{L}$ & ACG 105 & ICG 7906 & fastigiata & vulgaris & $\mathrm{L}$ \\
\hline ACG 51 & ICG 3775 & fastigiata & vulgaris & $\mathrm{L}$ & ACG 106 & ICG 7963 & hypogaea & hypogaea & $\mathrm{L}$ \\
\hline ACG 52 & ICG 3992 & hypogaea & hypogaea & $\mathrm{S}$ & ACG 107 & ICG 7969 & fastigiata & vulgaris & $\mathrm{L}$ \\
\hline ACG 53 & ICG 4156 & hypogaea & hypogaea & $\mathrm{S}$ & ACG 108 & ICG 8083 & fastigiata & vulgaris & $\mathrm{L}$ \\
\hline ACG 54 & ICG 4343 & hypogaea & hypogaea & $\mathrm{S}$ & ACG 109 & ICG 8106 & fastigiata & fastigiata & $\mathrm{L}$ \\
\hline ACG 55 & ICG 4389 & hypogaea & hypogaea & $\mathrm{S}$ & ACG 110 & ICG 8285 & hypogaea & hypogaea & $\mathrm{S}$ \\
\hline
\end{tabular}


Table 1 continued

\begin{tabular}{|c|c|c|c|c|c|c|c|c|c|}
\hline $\begin{array}{c}\text { Accession } \\
\text { No. }\end{array}$ & $\begin{array}{c}\text { ICRISAT } \\
\text { Genebank } \\
\text { Entry (ICG) / } \\
\text { Cultivar } \\
\text { Name }\end{array}$ & Subspecies & $\begin{array}{c}\text { Botanical } \\
\text { variety }\end{array}$ & $\begin{array}{l}\text { Marker } \\
\text { Score* }\end{array}$ & Accession No. & $\begin{array}{c}\text { ICRISAT } \\
\text { Genebank } \\
\text { Entry (ICG) / } \\
\text { Cultivar } \\
\text { Name }\end{array}$ & Subspecies & $\begin{array}{c}\text { Botanical } \\
\text { variety }\end{array}$ & $\begin{array}{c}\text { Marker } \\
\text { Score }\end{array}$ \\
\hline ACG 111 & ICG 8490 & hypogaea & hypogaea & $\mathrm{S}$ & ACG 166 & ICG 13858 & fastigiata & fastigiata & $\mathrm{L}$ \\
\hline ACG 112 & ICG 8517 & fastigiata & fastigiata & $\mathrm{L}$ & ACG 167 & ICG 13941 & fastigiata & vulgaris & $\mathrm{S}$ \\
\hline ACG 113 & ICG 8567 & fastigiata & vulgaris & $\mathrm{L}$ & ACG 168 & ICG 13942 & hypogaea & hypogaea & $\mathrm{S}$ \\
\hline ACG 114 & ICG 8760 & hypogaea & hypogaea & $\mathrm{S}$ & ACG 169 & ICG 13982 & hypogaea & hypogaea & $\mathrm{L}$ \\
\hline ACG 115 & ICG 9037 & hypogaea & hypogaea & $\mathrm{S}$ & ACG 170 & ICG 14008 & hypogaea & hypogaea & $\mathrm{S}$ \\
\hline ACG 116 & ICG 9157 & fastigiata & vulgaris & $\mathrm{L}$ & ACG 171 & ICG 14106 & fastigiata & fastigiata & $\mathrm{L}$ \\
\hline ACG 117 & ICG 9249 & fastigiata & vulgaris & $\mathrm{L}$ & ACG 172 & ICG 14118 & fastigiata & vulgaris & $\mathrm{L}$ \\
\hline ACG 118 & ICG 9315 & fastigiata & fastigiata & $\mathrm{L}$ & ACG 173 & ICG 14127 & fastigiata & fastigiata & $\mathrm{L}$ \\
\hline ACG 119 & ICG 9418 & fastigiata & vulgaris & $\mathrm{L}$ & ACG 174 & ICG 14466 & hypogaea & hypogaea & $\mathrm{S}$ \\
\hline ACG 120 & ICG 9507 & fastigiata & vulgaris & $\mathrm{S}$ & ACG 175 & ICG 14475 & hypogaea & hypogaea & $\mathrm{L}$ \\
\hline ACG 121 & ICG 9666 & hypogaea & hypogaea & $\mathrm{S}$ & ACG 176 & ICG 14482 & hypogaea & hypogaea & $\mathrm{L}$ \\
\hline ACG 122 & ICG 9777 & hypogaea & hypogaea & $\mathrm{S}$ & ACG 177 & ICG 14523 & hypogaea & hypogaea & $\mathrm{S}$ \\
\hline ACG 123 & ICG 9809 & fastigiata & vulgaris & $\mathrm{L}$ & ACG 178 & ICG 14630 & fastigiata & fastigiata & $\mathrm{L}$ \\
\hline ACG 124 & ICG 9842 & hypogaea & hypogaea & $\mathrm{S}$ & ACG 179 & ICG 14705 & hypogaea & hypogaea & $\mathrm{L}$ \\
\hline ACG 125 & ICG 9905 & hypogaea & hypogaea & $\mathrm{S}$ & ACG 180 & ICG 14710 & fastigiata & fastigiata & $\mathrm{L}$ \\
\hline ACG 126 & ICG 9961 & hypogaea & hypogaea & $\mathrm{S}$ & ACG 181 & ICG 14985 & fastigiata & vulgaris & $\mathrm{L}$ \\
\hline ACG 127 & ICG 10036 & fastigiata & peruviana & $\mathrm{L}$ & ACG 182 & ICG 15042 & fastigiata & fastigiata & $\mathrm{L}$ \\
\hline ACG 128 & ICG 10092 & fastigiata & fastigiata & $\mathrm{L}$ & ACG 183 & ICG 15190 & hypogaea & hypogaea & $\mathrm{S}$ \\
\hline ACG 129 & ICG 10185 & hypogaea & hypogaea & $\mathrm{S}$ & ACG 184 & ICG 15287 & fastigiata & vulgaris & $\mathrm{L}$ \\
\hline ACG 130 & ICG 10384 & fastigiata & vulgaris & $\mathrm{L}$ & ACG 185 & ICG 15309 & fastigiata & fastigiata & $\mathrm{L}$ \\
\hline ACG 131 & ICG 10474 & fastigiata & fastigiata & $\mathrm{L}$ & ACG 186 & ICG 15419 & hypogaea & hirsuta & $\mathrm{L}$ \\
\hline ACG 132 & ICG 10479 & hypogaea & hypogaea & $\mathrm{L}$ & ACG 187 & $\mathrm{NC}-7$ & hypogaea & hypogaea & $\mathrm{S}$ \\
\hline ACG 133 & ICG 10554 & fastigiata & fastigiata & $\mathrm{L}$ & ACG 188 & PF-259860 & hypogaea & hypogaea & $\mathrm{S}$ \\
\hline ACG 134 & ICG 10566 & fastigiata & fastigiata & $\mathrm{L}$ & ACG 189 & NC-3033 & hypogaea & hypogaea & $\mathrm{S}$ \\
\hline ACG 135 & ICG 10890 & fastigiata & fastigiata & $\mathrm{L}$ & ACG 190 & 5015 & hypogaea & hypogaea & $\mathrm{S}$ \\
\hline ACG 136 & ICG 11088 & fastigiata & peruviana & $\mathrm{L}$ & ACG 191 & 5026 & hypogaea & hypogaea & $\mathrm{S}$ \\
\hline ACG 137 & ICG 11109 & hypogaea & hypogaea & $\mathrm{L}$ & ACG 192 & 5030 & hypogaea & hypogaea & $\mathrm{L}$ \\
\hline ACG 138 & ICG 11144 & fastigiata & fastigiata & $\mathrm{L}$ & ACG 193 & 5067 & hypogaea & hypogaea & $\mathrm{L}$ \\
\hline ACG 139 & ICG 11219 & hypogaea & hypogaea & $\mathrm{S}$ & ACG 194 & $88 / 3$ & hypogaea & hypogaea & $\mathrm{S}$ \\
\hline ACG 140 & ICG 11249 & fastigiata & vulgaris & $\mathrm{L}$ & ACG 195 & Ant-92/1 & hypogaea & hypogaea & $\mathrm{S}$ \\
\hline ACG 141 & ICG 11322 & hypogaea & hypogaea & $\mathrm{S}$ & ACG 196 & $427-24$ & hypogaea & hypogaea & $\mathrm{S}$ \\
\hline ACG 142 & ICG 11426 & hypogaea & hypogaea & $\mathrm{L}$ & ACG 197 & $437-3-4-B-2$ & hypogaea & hypogaea & $\mathrm{L}$ \\
\hline ACG 143 & ICG 11457 & hypogaea & hypogaea & $\mathrm{S}$ & ACG 198 & $393-2-1-2-2$ & hypogaea & hypogaea & $\mathrm{N} / \mathrm{A}$ \\
\hline ACG 144 & ICG 11515 & fastigiata & vulgaris & $\mathrm{L}$ & ACG 199 & $70 / 1145-1 / 03$ & hypogaea & hypogaea & $\mathrm{L}$ \\
\hline ACG 145 & ICG 11651 & fastigiata & vulgaris & $\mathrm{L}$ & ACG 200 & 75/1073-A & hypogaea & hypogaea & $\mathrm{S}$ \\
\hline ACG 146 & ICG 11687 & fastigiata & vulgaris & $\mathrm{L}$ & ACG 201 & 75/1073-B & hypogaea & hypogaea & $\mathrm{S}$ \\
\hline ACG 147 & ICG 11855 & hypogaea & hypogaea & $\mathrm{S}$ & ACG 202 & Bari-89 & hypogaea & hypogaea & $\mathrm{S}$ \\
\hline ACG 148 & ICG 11862 & hypogaea & hypogaea & $\mathrm{S}$ & ACG 203 & Best Dagar & hypogaea & hypogaea & N/A \\
\hline ACG 149 & ICG 12000 & hypogaea & hypogaea & $\mathrm{S}$ & ACG 204 & V.Banbim P. & hypogaea & hypogaea & $\mathrm{S}$ \\
\hline ACG 150 & ICG 12189 & fastigiata & vulgaris & $\mathrm{L}$ & ACG 205 & 88 Bocounba & hypogaea & hypogaea & $\mathrm{S}$ \\
\hline ACG 151 & ICG 12276 & hypogaea & hypogaea & $\mathrm{L}$ & ACG 206 & Home bay & hypogaea & hypogaea & $\mathrm{S}$ \\
\hline ACG 152 & ICG 12370 & hypogaea & hypogaea & $\mathrm{S}$ & ACG 207 & Florigiant & hypogaea & hypogaea & $\mathrm{L}$ \\
\hline ACG 153 & ICG 12625 & fastigiata & aequatoriana & $\mathrm{L}$ & ACG 208 & Flamingo & hypogaea & hypogaea & $\mathrm{S}$ \\
\hline ACG 154 & ICG 12672 & hypogaea & hypogaea & $\mathrm{S}$ & ACG 209 & Shulamit & hypogaea & hypogaea & $\mathrm{S}$ \\
\hline ACG 155 & ICG 12682 & fastigiata & vulgaris & $\mathrm{L}$ & ACG 210 & Sunrunner & hypogaea & hypogaea & $\mathrm{S}$ \\
\hline ACG 156 & ICG 12697 & fastigiata & vulgaris & N/A & ACG 211 & Florunner & hypogaea & hypogaea & $\mathrm{S}$ \\
\hline ACG 157 & ICG 12879 & fastigiata & vulgaris & $\mathrm{L}$ & ACG 212 & Swallow & hypogaea & hypogaea & $\mathrm{S}$ \\
\hline ACG 158 & ICG 12921 & fastigiata & vulgaris & $\mathrm{L}$ & ACG 213 & Behirim & hypogaea & hypogaea & $\mathrm{L}$ \\
\hline ACG 159 & ICG 12988 & fastigiata & vulgaris & $\mathrm{L}$ & ACG 214 & Cine & hypogaea & hypogaea & $\mathrm{S}$ \\
\hline ACG 160 & ICG 13099 & hypogaea & hypogaea & $\mathrm{S}$ & ACG 215 & Kadriye & hypogaea & hypogaea & $\mathrm{S}$ \\
\hline ACG 161 & ICG 13491 & fastigiata & vulgaris & $\mathrm{L}$ & ACG 216 & Osmaniye & hypogaea & hypogaea & N/A \\
\hline ACG 162 & ICG 13603 & fastigiata & vulgaris & $\mathrm{L}$ & ACG 217 & Osm. Erzin & hypogaea & hypogaea & $\mathrm{S}$ \\
\hline ACG 163 & ICG 13723 & hypogaea & hypogaea & $\mathrm{S}$ & ACG 218 & Anamur-B & hypogaea & hypogaea & $\mathrm{S}$ \\
\hline ACG 164 & ICG 13787 & hypogaea & hypogaea & $\mathrm{S}$ & ACG 219 & Anamur-K & hypogaea & hypogaea & $\mathrm{S}$ \\
\hline ACG 165 & ICG 13856 & fastigiata & fastigiata & $\mathrm{L}$ & ACG 220 & Gazipasa & hypogaea & hypogaea & $\mathrm{S}$ \\
\hline
\end{tabular}


Table 1 continued

\begin{tabular}{|c|c|c|c|c|c|c|c|c|c|}
\hline $\begin{array}{c}\text { Accession } \\
\text { No. }\end{array}$ & $\begin{array}{c}\text { ICRISAT } \\
\text { Genebank } \\
\text { Entry (ICG) / } \\
\text { Cultivar } \\
\text { Name }\end{array}$ & Subspecies & $\begin{array}{c}\text { Botanical } \\
\text { variety }\end{array}$ & $\begin{array}{l}\text { Marker } \\
\text { Score* }\end{array}$ & Accession No. & $\begin{array}{c}\text { ICRISAT } \\
\text { Genebank } \\
\text { Entry (ICG) / } \\
\text { Cultivar } \\
\text { Name }\end{array}$ & Subspecies & $\begin{array}{c}\text { Botanical } \\
\text { variety }\end{array}$ & $\begin{array}{c}\text { Marker } \\
\text { Score }\end{array}$ \\
\hline ACG 221 & Çom & hypogaea & hypogaea & $\mathrm{S}$ & ACG 239 & Schwarz & fastigiata & fastigiata & $\mathrm{L}$ \\
\hline ACG 222 & NC-Fla-14 & hypogaea & hypogaea & $\mathrm{S}$ & ACG 240 & Spancross & fastigiata & fastigiata & $\mathrm{L}$ \\
\hline ACG 223 & NC-10-C & hypogaea & hypogaea & $\mathrm{S}$ & ACG 241 & PF-161317 & fastigiata & fastigiata & $\mathrm{L}$ \\
\hline ACG 224 & GP-NC-343 & hypogaea & hypogaea & $\mathrm{S}$ & ACG 242 & PF-248759 & fastigiata & fastigiata & $\mathrm{L}$ \\
\hline ACG 225 & 88488 & hypogaea & hypogaea & $\mathrm{S}$ & ACG 243 & PF-268771-B & fastigiata & fastigiata & $\mathrm{L}$ \\
\hline ACG 226 & 88121 & hypogaea & hypogaea & $\mathrm{S}$ & ACG 244 & C $1-27$ & fastigiata & fastigiata & $\mathrm{L}$ \\
\hline ACG 227 & PI-315633 & hypogaea & hypogaea & $\mathrm{S}$ & ACG 245 & AF-2B Grif & fastigiata & fastigiata & $\mathrm{L}$ \\
\hline ACG 228 & PI-315621 & hypogaea & hypogaea & $\mathrm{S}$ & ACG 246 & Argentine & fastigiata & fastigiata & $\mathrm{L}$ \\
\hline ACG 229 & Edirne-9p-53 & hypogaea & hypogaea & $\mathrm{S}$ & ACG 247 & Bayramic & fastigiata & fastigiata & $\mathrm{L}$ \\
\hline ACG 230 & M-44-A & hypogaea & hypogaea & $\mathrm{S}$ & ACG 248 & Comet & fastigiata & fastigiata & $\mathrm{L}$ \\
\hline ACG 231 & M-44-B & hypogaea & hypogaea & $\mathrm{L}$ & ACG 249 & N. M.Valan & fastigiata & fastigiata & $\mathrm{L}$ \\
\hline ACG 232 & Anamur-2006 & hypogaea & hypogaea & $\mathrm{L}$ & ACG 250 & T. Power & fastigiata & fastigiata & $\mathrm{L}$ \\
\hline ACG 233 & 97-Vietname & fastigiata & fastigiata & $\mathrm{L}$ & ACG 251 & 96-Australia & fastigiata & fastigiata & $\mathrm{L}$ \\
\hline ACG 234 & 98-Australia & fastigiata & fastigiata & $\mathrm{S}$ & ACG 252 & Taianan & fastigiata & fastigiata & $\mathrm{S}$ \\
\hline ACG 235 & Florispan & fastigiata & fastigiata & $\mathrm{L}$ & ACG 253 & $\mathrm{H}$ & fastigiata & fastigiata & $\mathrm{S}$ \\
\hline ACG 236 & 1 & fastigiata & fastigiata & $\mathrm{L}$ & ACG 254 & Early rumir & fastigiata & fastigiata & $\mathrm{L}$ \\
\hline ACG 237 & $18 / 38$ & fastigiata & fastigiata & $\mathrm{L}$ & ACG 255 & Egret & fastigiata & fastigiata & $\mathrm{S}$ \\
\hline ACG 238 & Starr & fastigiata & fastigiata & $\mathrm{L}$ & ACG 256 & DixilAnax & fastigiata & fastigiata & $\mathrm{S}$ \\
\hline
\end{tabular}

$* L$ is resistant, $S$ is susceptible, $N / A$ is no amplification

$10 \times$ PCR buffer, $0.4 \mathrm{mM}$ of dNTPs mix, $2.5 \mathrm{mM}$ of $\mathrm{MgCl}_{2}, 0.3 \mu \mathrm{M}$ each primer, 1 unit of Taq DNA polymerase (Fermentas Life Sciences, Burlington, Canada), $1 \mu \mathrm{l}$ genomic DNA template and Milli-Q water to a final volume of $20 \mu \mathrm{l}$. The SSR marker (Forward primer: 5' TACAGCATTGCCTTCTGGTG 3'; Reverse primer: 5' GCACACCATGGCTCAGTTATT $3^{\prime}$ ), tightly linked to sclerotinia blight resistance gene (Chenault et al. 2009), amplification was performed in a programmable thermocycler (BIONEER, MyGenie ${ }^{\mathrm{TM}}$ ) under the following conditions: $94{ }^{\circ} \mathrm{C}$ for 2 min, 35 cycles of $94{ }^{\circ} \mathrm{C}$ for $45 \mathrm{~s}$, annealing temperature $60{ }^{\circ} \mathrm{C}$ for $1 \mathrm{~min}, 72{ }^{\circ} \mathrm{C}$ for $90 \mathrm{~s}$, and then a final extension of $10 \mathrm{~min}$ at $72{ }^{\circ} \mathrm{C}$ (Ferguson et al. 2004). PCR products were separated in 2-3\% agarose gels in $1 \times$ TBE buffer and visualized under UV light after staining with ethidium bromide. The expected bands were determined visually and recorded. Amplified products were also analyzed in the Fragment Analyzer $^{\mathrm{TM}}$ which is high resolution bio-imaging system (Advanced Analytical Technologies GmbH, Heidelberg, Germany). The DNF-900 Reagent Kit was used for qualitative analysis of DNA fragments ranging from 35 to $500 \mathrm{bp}$. The markers for 35 and $500 \mathrm{bp}$ fragments were used for normalization, respectively. After analysis, virtual gel imaging was analyzed with the software PROSize 2.0 (Version 1.2.1.1) (Advanced Analytical Technologies, AMES, IA, USA). All reactions were performed twice. Amplified bands were scored as previously reported by Chenault et al. (2009). The authors identified four possible band amplifications ( $\mathrm{L}, \mathrm{S}, \mathrm{B}$, and $\mathrm{b}$ ) using with the sclerotinia blight associated marker. If a genotype had only the $145 \mathrm{bp}$ band, it was scored as L. When genotypes had predominant 145 and $100 \mathrm{bp}$ bands, they were scored as B and b, respectively. Genotypes amplified only $100 \mathrm{bp}$ band were given an S score.

\section{Results and discussion}

Molecular marker analysis was performed to screen sclerotinia blight resistance in groundnut collection. Two different band patterns ( $\mathrm{L}$ and $\mathrm{S}$ ) were observed in agarose gel (Fig. 1) and high bio-imaging system following to PCR amplification (Fig. 2). No genotypes had patterns $B$ and $b$.

Chenault et al. (2009) stated that genotypes with $145 \mathrm{bp}$ band were scored as "L" associated with sclerotinia blight resistance while genotypes carrying only $100 \mathrm{bp}$ were given a score of " $\mathrm{S}$ " indicating 


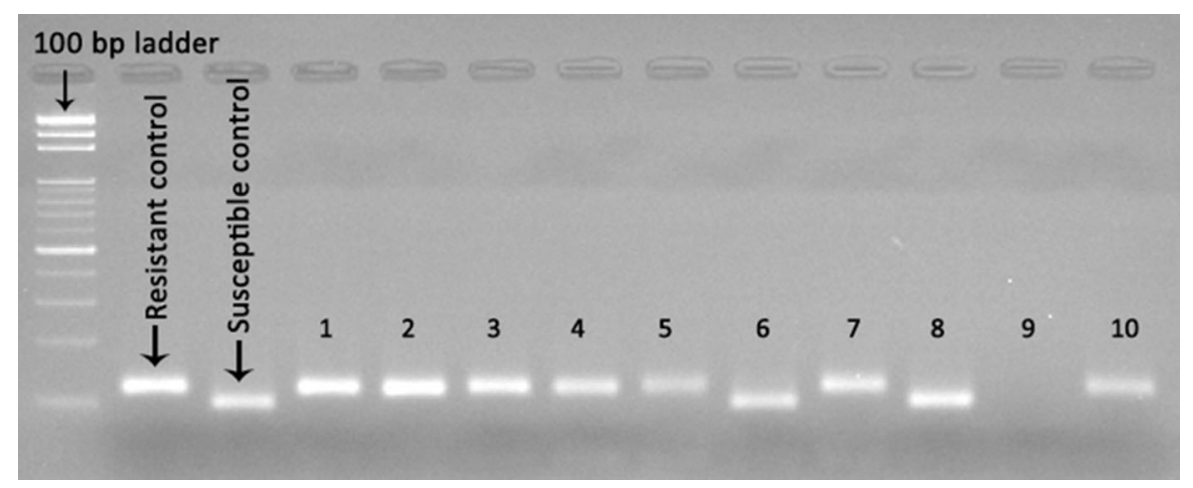

Fig. 1 Agarose gel showing the amplification products using sclerotinia blight associated marker. Resistant and susceptible controls are PI 482189 and PI 496448, respectively. The selected genotypes are numbered as follows: lanes 1-10, ACG 1; ACG 9; ACG 24; ACG 25; ACG 30; ACG 38; ACG 127; ACG 122; ACG 91; ACG 207
Fig. 2 Fragment

Analyzer ${ }^{\mathrm{TM}}$ shows the gel picture and peak analysis graphic for the selected resistant/susceptible genotypes
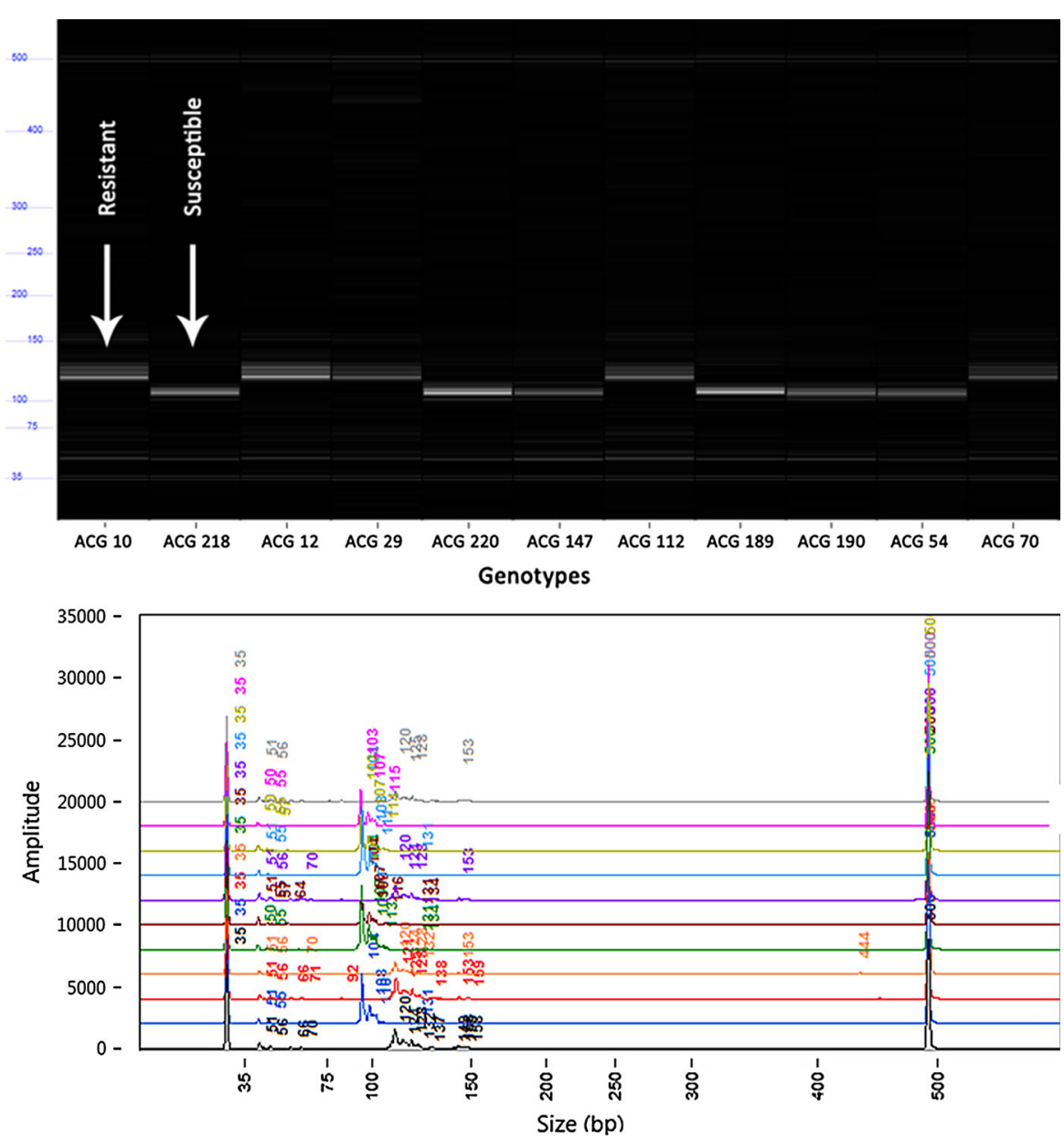

susceptibility. In the present study, 142 genotypes showed the sclerotinia blight resistant fragment with $145 \mathrm{bp}$ in the collection, while $100 \mathrm{bp}$ band detected in 108 genotypes was associated with susceptibility
(Table 1). Rest of six genotypes amplified no band following PCR amplification. The resistance allele in this study was present in higher frequency than susceptible resistance allele in the groundnut 
Table 2 Botanical variety comparison of genotypes tested by the marker

\begin{tabular}{lrccr}
\hline $\begin{array}{l}\text { Botanical } \\
\text { variety }\end{array}$ & \multicolumn{4}{l}{ Marker score } \\
\cline { 2 - 5 } & L & S & B & b \\
\hline hypogaea & 27 & 101 & - & - \\
fastigiata & 54 & 5 & - & - \\
vulgaris & 56 & 3 & - & - \\
peruviana & 2 & - & - & - \\
aequatoriana & 1 & - & - & - \\
hirsuta & 1 & - & - & - \\
\hline
\end{tabular}

collection. The present investigation therefore reports new sources of resistance to sclerotinia blight in groundnut. For a better comparison, in the U.S peanut mini core collection only 39 individuals from spanish, valencia, runner market types were classified as new potential sources of resistance (Chamberlin et al. 2010).

The cultivated groundnut has two subspecies, $A$. hypogaea ssp. hypogaea and A. hypogaea ssp. fastigiata (Gregory et al. 1980), and six botanical varieties hypogaea, hirsuta, fastigiata, peruviana, aequuatoriana and vulgaris. Moreover, the commercially grown cultivars are grouped into four market classes, the runner, virginia, spanish, and valencia market types (Krapovickas and Rigoni 1994). In the present study, the vulgaris types (39.4\%) were found more resistant to sclerotinia blight than those of fastigiata (38.0\%) and hypogaea (19.7\%) types (Table 2), with showing $\mathrm{R}$ banding pattern using the SSR marker among the resistant genotypes of the collection. This result highly compatible with the field study by Porter et al. (1975) who stated that spanish-type (variety vulgaris) groundnut has more resistance to sclerotinia blight than virginia types. Chenault et al. (2009) also identified high resistance in spanish types through molecular analysis.

This study included 186 groundnut mini core genotypes from ICRISAT. In the mini core collection, the $\mathrm{R}$ banding pattern related to the disease resistance is observed at high frequency in the variety vulgaris $(48.6 \%)$ and less distributed among the varieties fastigiata $(31.3 \%)$ and hypoegaea $(17.3 \%)$ among the resistant genotypes in the mini-core collection. Variety peruviana had only two genotypes in the collection and both of them consistent with resistance marker. The botanical variety aequatoriana was presented only one germplasm which was indicated resistance to sclerotinia blight disease after molecular analysis. The presence of sclerotinia blight resistance in the U.S. peanut mini core collection was also examined with molecular survey by Chamberlin et al. (2010). All spanish genotypes in that collection carrying $\mathrm{S}$ banding pattern which is less observed in the virginia (46\%) and runner market types (35\%). These findings confirm the usefulness of the sclerotinia blight resistant marker and effectiveness of MAS for groundnut breeding programs.

The wild species are good sources of sclerotinia blight resistance genes in groundnut (Tallury et al. 2014). However interspecific incompatibility and unfavorable linkage drag associated with resistance to diseases cause to limited success in transferring disease resistance (Murty and Jahnavi 1983). Molecular markers tightly linked to disease resistance loci may increase selection efficiency in interspecific derivatives (Mace et al. 2006). In this study, the disease resistant marker indicated that it could be directly used for marker-assisted breeding. However it is insufficient without yield traits. Groundnut is an industrial crop and comprehensive information or database on agronomic traits of all genotypes must be available for optimal commercial exploitation. The sclerotinia blight resistant sources identified from different botanical varieties in the present study therefore should be evaluated agronomically to provide better opportunities in developing high yielding resistant cultivars appropriate for different regions.

Acknowledgments This study was supported by the Ministry of Science, Industry and Technology of Turkey with the grant number of SANTEZ-01527-STZ-2012-2. We are grateful to International Crops Research Institute for the Semi-Arid Tropics (ICRISAT), Gene bank, Hyderabad, India for supplying genetic material and to the Scientific Research Projects Coordination Unit of Akdeniz University for continuous supports.

Conflict of interest The authors declare that there are no conflicts of interest.

\section{References}

Arus P, Moreno-Gonzalez J (1993) Marker-assisted selection. In: Hayward MD, Bosemark NO, Romagosa I (eds) Plant breeding: principles and prospects. Chapman and Hall, Cambridge, pp 315-331 
Ashkani S, Rafii MY, Rusli I, Sariah M, Abdullah SNA, Rahim HA, Latif MA (2012) SSRs for marker-assisted selection for blast resistance in rice (Oryza sativa $\mathrm{L}$.). Plant Mol Biol Report 30:79-86

Bailey JE, Brune PD (1997) Effect of crop pruning on Sclerotinia blight of peanut. Plant Dis 81:990-995

Boopathi NM (2013) Genetic mapping and marker assisted selection. Springer, India

Butzler TM, Bailey J, Beute MK (1998) Integrated management of Sclerotinia blight in peanut: utilizing canopy morphology, mechanical pruning, and fungicide timing. Plant Dis 82:1312-1318

Chamberlin KDC, Melouk HA, Payton ME (2010) Evaluation of the U.S. peanut mini core collection using a molecular marker for resistance to Sclerotinia minor Jagger. Euphytica 172:109-115

Chenault KD, Maas A, Melouk HA, Payton ME (2009) Discovery and characterization of a molecular marker for Sclerotinia minor (Jagger) resistance in peanut. Euphytica 166:357-365

Doyle JJ, Doyle JL (1990) A rapid total DNA preparation procedure for fresh plant tissue. Focus 12:13-15

Ender M, Terpstra K, Kelly JD (2008) Marker-assisted selection for white mold resistance in common bean. Mol Breed 21:149-157

FAO (2012) FAOSTAT. FAO, Rome. http://faostat.fao.org/site/ 567/default.aspx. Accessed 18 June 2014

Ferguson ME, Burow MD, Schulze SR, Bramel PJ, Paterson AH, Kresovich S, Mitchell S (2004) Microsatellite identification and characterization in peanut (Arachis hypogaea L.). Theor Appl Genet 108:1064-1070

Gil SV, Harob R, Oddinoc C, Kearneyc M, Zuzac M, Marinellic A, Marcha GJ (2008) Crop management practices in the control of peanut diseases caused by soilborne fungi. Crop Prot 27:1-9

Gregory W, Krapovickas A, Gregory M (1980) Structure, variation, evolution, and classification in Arachis. In: Summerfield R, Bunting A (eds) Advances in legume science. Royal Botanic Gardens, Kew, London, pp 469-481

Krapovickas SA, Rigoni VA (1994) Taxonomia del genero Arachis (Leguminosae). Bonplandia 8:1-186

Laemmlen F (2001) Sclerotinia diseases. Agriculture and Natural Resources, University of California Publication, 8042:1-5

Liao B, Holbrook C (2007) Groundnut. In: Singh RJ (ed) Genetics resources, chromosome engineering and crop improvement, Oilseed Crops, vol 4. CRC Press, Boca Raton, pp 231-289

Livingstone DM, Hampton JL, Phipps PM, Grabau EA (2005) Enhancing resistance to Sclerotinia minor in peanut by expressing a barley oxalate oxidase gene. Plant Physiol 137:1354-1362

Mace ES, Phong DT, Upadhyaya HD, Chandra S, Crouch JH (2006) SSR analysis of cultivated groundnut (Arachis hypogaea L.) germplasm resistant to rust and late leaf spot diseases. Euphytica 152:317-330
Melouk HA, Backman PA (1995) Management of soil borne fungal pathogens. In: Melouk HA, Shokes FM (eds) Peanut health management. APS, Minnesota, pp 75-82

Mondal S, Badigannavar AM (2010) Molecular diversity and association of SSR markers to rust and late leaf spot resistance in cultivated groundnut (Arachis hypogaea L.). Plant Breed 129:68-71

Murty UR, Jahnavi MR (1983) Breeding potential of interspecific tetraploids in Arachis L. In: Feakin SD (ed) Proceedings of an international workshop on cytogenetics of Arachis. ICRISAT, Patancheru, pp 125-130

Nagy ED, Chu Y, Guo Y, Khanal S, Tang S, Li Y, Dong W, Timper P, Taylor C, Ozias-Akins P, Holbrook CC, Beilinson V, Nielsen NC, Stalker HT, Knapp SJ (2010) Recombination is suppressed in an alien introgression on chromosome $5 \mathrm{~A}$ of peanut harboring Rma, a dominant root knot nematode resistance gene. Mol Breed 26:357-370

Nigam SN, Aruna R (2008) Improving breeding efficiency for early maturity in peanut. Plant Breed Rev 30:295-316

Porter DM, Melouk HM (1997) Sclerotinia blight. In: KokalisBurelle N, Porter DM, Rodriguez-Kabana R, Smith DH, Subrahmanyam P (eds) Compendium of peanut diseases, 2nd edn. American Phytopathological Society Press, St. Paul, pp 34-35

Porter DM, Beute MK, Wyne JC (1975) Resistance of peanut germplasm to Sclerotinia sclerotiorum. Peanut Sci 2:78-80

Sarvamangala C, Gowda MVC, Varshney RK (2011) Identification of quantitative trait loci for protein content, oil content and oil quality for groundnut (Arachis hypogaea L.). Field Crop Res 122:49-59

Shoba D, Manivannan N, Vindhiyavarman P, Nigam SN (2012) SSR markers associated for late leaf spot disease resistance by bulked segregant analysis in groundnut (Arachis hypogaea L.). Euphytica 188:265-272

Smith FD, Phipps PM, Stipes RJ (1992) Fluazinam: a new fungicide for control of sclerotinia blight and other soil borne diseases of peanut. Peanut Sci 19:115-120

Tallury SP, Hollowell JE, Isleib TG, Stalker HT (2014) Greenhouse evaluation of section Arachis wild species for sclerotinia blight and cylindrocladium black rot resistance. Peanut Sci 41:17-24

Thiessen LD, Woodward JE (2012) Diseases of peanut caused by soilborne pathogens in the Southwestern United States. ISRN Agron 2012:1-9

Upadhyaya HD, Bramel PJ, Ortiz R, Singh S (2002) Developing a mini core of peanut for utilization of genetic resources. Crop Sci 42:2150-2156

Upadhyaya HD, Sharma S, Dwivedi SL (2011) Arachis. In: Chittaranjan K (ed) Wild crop relatives: genomic and breeding resources legume crops and forages. Springer, Berlin, pp 1-19

Wu BM, Subbarao KV (2003) Effects of irrigation and tillage on the dynamics of Sclerotinia minor sclerotia and lettuce drop incidence. Phytopathology 93:1572-1580 